\title{
Expert Views on ... \\ ... the Future of Digital Media in Psychology
}

DOI 10.24989/dp.v1i1.1847

We asked three experts in the field about future perspectives on digital media in psychology and how it will affect our lives in the next decade. Jeremy Bailenson (Stanford University), Christian Montag (Ulm University) and Thomas Probst (Danube University of Krems) spoke to Digital Psychology [DP] about these topics.

[DP] How will digital media change the fields of psychology and psychological therapy in the next 5 years?

Thomas Probst: Digital media will transform mental health care and clinical research in the next 5 years. Internet-and mobilebased tools have the potential to globally disseminate evidencebased mental health interventions and to make diagnostic procedures more accurate. Research will move towards a more transdisciplinary approach in order to scientifically develop and evaluate digital media including all stakeholders.

Christian Montag: Naturally this is a hard question to answer, because we have already seen in recent years fast innovation cycles in digital related sciences causing disruptions in many areas of the global economy/societies (regardless of whether we speak of outdated concepts such as the "West" or the "East"). In so far five years are a long time to take a look into the crystal ball. Nevertheless, from what I observe in my research area, I am convinced that we will see a paradigm shift. The availability of digital footprints which can provide insights into a myriad of psychological traits and states and the underlying neurobiology is unprecedented. Therefore, I also foresee that the disciplines of psychology, medicine and computer science naturally will need to heighten their collaborative efforts - perhaps merging into a discipline called Psycho-Neuro-Informatics. Aside from this, I am also convinced that digital data layers present "only" an additional layer available to be studied by behavioral scientists. For instance, classic techniques such as self-report will always be of relevance if we want to understand how people feel or see themselves.

\section{[DP] What are the main challenges of using VR in research?}

Jeremy Bailenson: Research needs to engage larger sample sizes samples that are not simply comprised of university students examine repeated usage of VR over time, and look at longitudinal outcome measures.
[DP] What are the main challenges of using digital media in research and therapy?

Christian Montag: As we all know, Big Data comes in great variability concerning its variety (data formats), volume and velocity, which we refer to as the VVVs. And now comes the problem: Psychologists are used to carrying out descriptive and inferential statistics, which are still of relevance. But it is also true that when dealing with Big Data these more classic forms of data analysis need to be complemented by new analysis strategies, including machine learning. Beyond this methodological issue, the danger arises that researchers will more often detect spurious correlations which will not be meaningful, because they just appear at random. Therefore, it will be of utmost importance to bring theory guided thinking to the area of Big Data analysis. Of course, this does not mean that exploratory analyses are not valuable when examining Big Data. But such analyses have to be identified as being exploratory in nature and the findings need to be replicated.

Thomas Probst: Most of the digital media tools currently available in the fields of clinical psychology and psychotherapy are not rigorously tested in controlled trials. The challenge is to establish a quality assurance system for digital media that informs one about the digital tools' actual quality. In order to prevent harm, only scientifically-evaluated evidence-based digital tools shall be recommended. Other challenges include training therapists in effectively applying digital media as well as regulating who prescribes and covers the costs of their deployment.

[DP] What do you expect from mobile assessments, i-CBT, Virtual Reality, Digital Phenotyping and Big Data in the next decade? How will this affect therapeutic interventions?

Christian Montag: Predicting psychological traits/states from digital footprints produced as a result of a person's interaction with a coffee machine, the fridge or the smartphone in the Internet of Things (also called digital phenotyping or mobile sensing when done from mobile devices) will provide researchers with never before seen insights into human behavior. Crucially, such insights will be also gained on a longitudinal level. This said, I am aware that privacy concerns will dramatically increase in the coming years and it will be an important task, for both society as a whole and IRBs more specifically, to develop sound data 
protection plans. This is also necessary to protect the rights of participants in psychological studies in an age where researchers are increasingly asked to upload their complete (raw) data sets to repositories such as the Open Science Framework (OSF). Without doubt, frameworks such as the OSF have led to important improvements in the psychological sciences, and were a necessary response to the replication crisis in psychology. My group also supports this movement and uploads data when possible. But let's be honest: This also has the potential to exert tremendous costs on the participants-side of psychological studies. This is particularly pertinent if people participate in several studies, generating distinct data sets which could be matched by machine learning procedures. Here, important new research areas arise in psychology - namely studying privacy. Finally, an urgent discussion is required on what kind of (inferred) data can and should be used for what purposes in society? Should HR-departments be allowed to hire individuals according to their digital footprints? What about insurance companies using our digital data to set a price for policies and so forth? As our understanding of what digital traces can tell us about a person is increasing, we also currently face dramatic issues such as manipulation of elections. Should microtargeting based on digital footprints be banned in the context of elections? I am convinced that these challenges can only be solved with political regulation.

Jeremy Bailenson: What I would like to see in Virtual Reality and Augmented Reality research moving forward is methodological rigor that matches the recent availability and cost of hardware and content.

Thomas Probst: In the next decade, digital assessments of smart mobile devices will generate Big Data for digital phenotyping of mental health. This will produce totally novel scientific findings and data, and make precision-driven mental health care possible.
More and more therapists will integrate Virtual Reality Therapy, Internet- and mobile-based interventions (not restricted to iCBT), and smart digital diagnostics in their practical work. There is an urgent need to keep clinicians and consumers up-to-date about the opportunities and risks of currently available digital media. Governments must recognize the need to provide oversight and regulation to protect otherwise vulnerable consumers.

\section{Info Interviewees}

\section{Jeremy Bailenson}

Founding director of the Virtual Human Interaction Lab at Stanford University, US. Thomas More Storke Professor in the Department of Communication, Professor (by courtesy) of Education, Professor (by courtesy) Program in Symbolic Systems, a Senior Fellow at the Woods Institute for the Environment, and a Faculty Leader at Stanford's Center for Longevity.

E: bailenso@stanford.edu

\section{Christian Montag}

Full Professor and head of the department Molecular Psychology at Ulm University, Germany. Heisenberg-Professor for Molecular Psychology since 2014 at Ulm University, and since 2016 Agreement professor, University of Electronic Science and Technology of China (UESTC), Chengdu, China.

E: christian.montag@uni-ulm.de

\section{Thomas Probst}

Full Professor at the Department for Psychotherapy and Biopsychosocial Health of the Danube University Krems, Austria.

E: thomas.probst@donau-uni.ac.at 